\title{
A free-space alignment technique for active optical waveguide components
}

\author{
A. Karim \\ Department of Electronic Engineering, Sir Syed University of Engineering \& Technology, \\ University Road, Karachi 75300, Pakistan. \\ Phone: (92-21) 4988000-002,474583; fax. (92-21) 4982393; e-mail: abid@ssuet.edu.pk, akarimpk@yahoo.com
}

\begin{abstract}
In this paper an easy to use free-space alignment technique for the alignment of different active optical waveguide components is reported. This technique has successfully been implemented for the characterization of waveguide photodetectors with an intrinsic layer thickness of $0.15 \mu \mathrm{m}$. This technique is found to be very reliable and accurate.
\end{abstract}

Keywords: waveguide optical detector, active waveguide component.

Paper received 18.05.02; accepted for publication 10.12.02.

\section{Introduction}

It is well established that monolithic optoelectronic integrated circuits (OEICs), which incorporate electronic circuits and optical components (free-space or connected via optical interconnects or waveguides) on the same substrate, will play an important role in future data transmitting and processing systems. Due to their importance, OEICs are investigated extensively in order to take full advantage of optical means of data transmission and processing. Although monolithic integration is an ideal approach for the integration of several optical and electronic components on a single substrate, however, this approach is still a technological challenge. Large efforts are needed to solve the problems associated with monolithic approach step by step. In order to have a better understanding of light propagation in active waveguide components, a hybrid approach has been used as an intermediate step occasionally. The major advantage of a hybrid approach is that the performance of each component can be optimized according to a particular application. However hybrid integration suffers severely from the poor coupling of light from a source to a component. To overcome this problem, several passive and active alignment techniques have been suggested [1-4], but they all need sophisticated fabrication techniques for imple- mentation. Furthermore they are very much limited to a particular device geometry. Normally in a typical laboratory environment, butt-coupling alignment technique [5] and/or a free-space optical lens system with or without a tapered fiber is used to couple the light input to a component. In the butt-coupling technique, components have to be very close to each other; otherwise the technique suffers from beam divergence. If two components are placed very close to each other, temperature effects, which cause expansion of material, cannot be ignored. This could damage the facets of the device being used. Furthermore it becomes very difficult to isolate the laser source from reflection from other components. On the other hand, the tapered fiber technique also suffers from relatively high coupling losses of optical radiation. Nevertheless this technique has a reasonably large working distance and offers safety to the optical components from accidental facet damage. In both techniques, the alignment of different components is difficult, time consuming and may not be reliable. Here a reliable and easy to use technique for the alignment of active optical components is demonstrated by making use of optical radiation detection properties of a semiconductor laser diode. This technique is basically intended for a typical optoelectronic laboratory set-up where the different materials and components are needed to be characterized for future monolithic OEICs. 


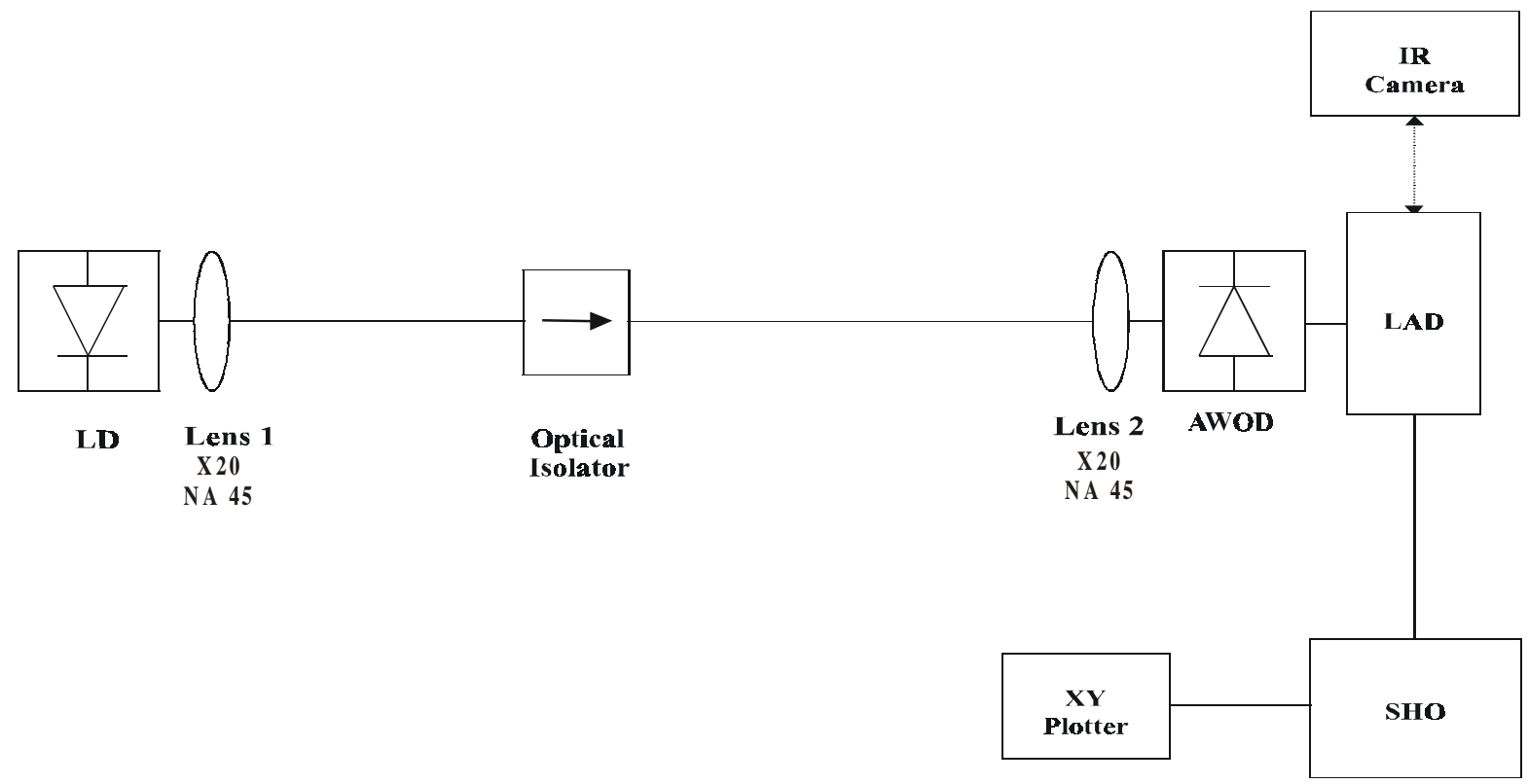

Fig. 1. Schematical representation of experimental set-up used for the implementation of the proposed alignment technique.

\section{Experimental procedure}

To demonstrate the validity of the proposed technique, two stripe geometry GaAs laser diodes with the active layer thickness of $0.15 \mu \mathrm{m}$ have been used (the device number-1 was used as the source laser and the device number- 2 was used as the active waveguide optical detector (AWOD) [6]). Both devices were mounted on separate heat sinks and a multi-channel temperature controller was used to control the temperature of both devices individually. The laser source was biased with $300 \mathrm{~ns}$ long pulses with a repetition frequency of $10 \mathrm{kHz}$ to avoid overheating in order to achieve more reliable results. Whereas AWOD was used under no bias conditions. A pre-calibrated large area detector (LAD) was used to measure light output and a infrared camera was used to observe near fields patterns of $L D$ and AWOD in place of $L A D$ as and when required. All the results were plotted using a sample \& hold oscilloscope and an X-Y plotter. Fig. 1 shows the schematic of experimental set-up used to implement this alignment technique.

First of all AWOD being investigated and the laser source were aligned with the help of IR camera in such a way that it was obstructing the optical path between the source laser and IR camera. By manipulating the position of the source laser, lens-1, lens-2 and AWOD and observing the near field beam profile of the source laser on the camera, a slight response of the AWOD was observed which was maximized by repositioning the input beam on to the input facet of AWOD. This maximum output of AWOD was representing the maximum alignment between the two active components. In order to check the reliability of alignment between the source laser and AWOD, the device-1 was used as an AWOD (under no bias condition) and the device- 2 as a source laser. The response of device-1 as an AWOD was found maximum at a point where the response of device-2 (used as AWOD) was maximum (in this case no optical isolator was used).

\section{Response of AWOD}

The response of an AWOD was analyzed by measuring the I-L characteristic of the source laser using device-2 as an AWOD. Also the I-L characteristic of the source laser was measured by placing LAD just after lens-2 (in place of AWOD). The results of both measurements are given in Fig. 2. Fig. 2 shows the validity of this technique owing to the fact that the response of a pre-calibrated $\mathrm{Si}$ LAD and the GaAs AWOD (being used) are similar to each other except the sensitivity. Fig. 3 shows the relationship between the input optical power to AWOD and the photocurrent through AWOD. From Fig. 3, the sensitivity of the AWOD was calculated to be $0.22 \mathrm{~A} / \mathrm{W}$. This value is well within the reported value of $[7,8]$.

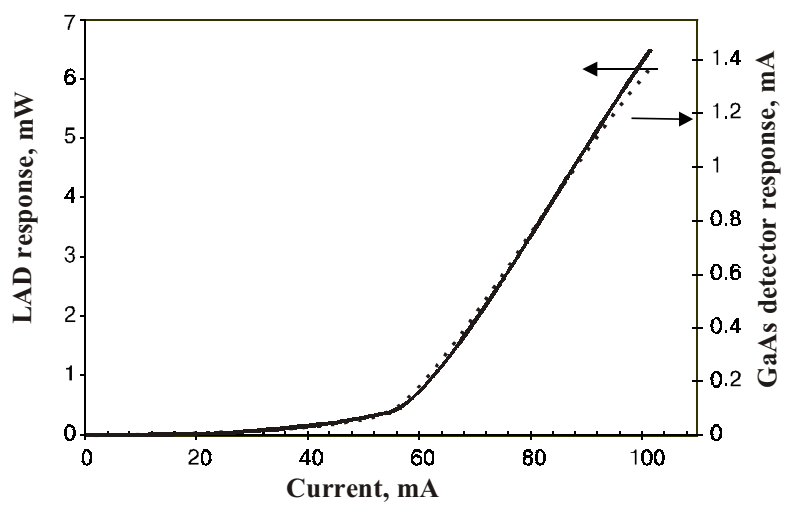

Fig. 2. Measured I-L characteristics for LAD and GaAs AWOD. 
Abid Karim: A free-space alignment technique for active optical waveguide components

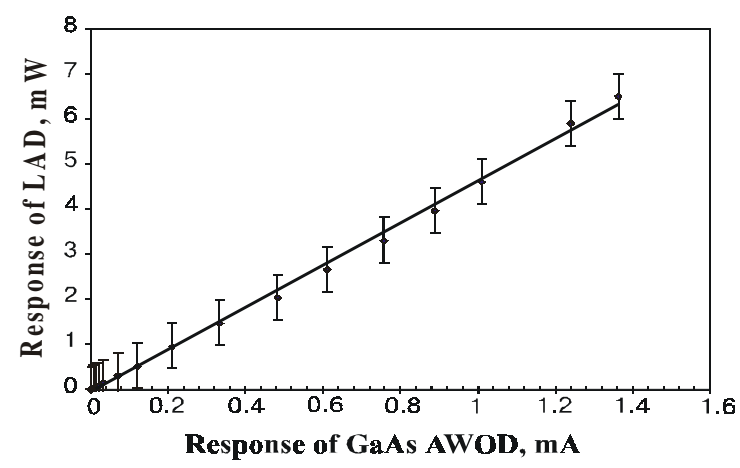

Fig. 3. Curve used to calculate the sensitivity of a GaAs AWOD.

\section{Conclusion}

In this paper an active alignment technique for active waveguide components for future free-space optoelectronic integrated circuit is demonstrated and implemented successfully.

The accuracy o this technique is expected to be in sub-micron region due to the fact that the thickness of the active regions of both devices was $0.15 \mu \mathrm{m}$ and slight misalignment would definitely have reduced the amount of detected power. Although this technique has been implemented on GaAs based devices, yet this technique can be used for the alignment of any active device.

\section{Acknowledgement}

The work reported in this letter was carried out at Sir Syed University of Engineering \& Technology (SSUET), Karachi, Pakistan. The author would like to acknowledge the support and contribution of Prof. Dr. Syed Enamul Haque, Dean Faculty of Engineering at SSUET.

\section{References}

1. Dagenais, M., Saini, S. S., Fan, Z. F., Seiferth, F., and Merritt, M. Complex needs drive optoelectronic integartion // Laser Focus, 34(7), 157-161 (1998).

2. Nakagawa, G., Yamamoto, T., Sasaki, S., Norimatsu, M., Yamamoto, N., Nosaka, T., Terada, K., Tanaka, K., Miura, K., and Yano, M. High power high sensitivity planar lightwave circuit module incorporating a novel passive alignment method // Journal of Lightwave Technol. 16(1), 66-72 (1998).

3. Graf, M. Active alignment process reduces fiber-core offsets // Laser Focus World, 32(7), 125-127 (1996).

4. Boisset, G. C., Robertson, B., and Hinton, H. S. Design and construction of active demonstrator for free-space optical interconnect // IEEE Photonic Technology Lett., 7(6), 676678 (1995).

5. Karim, A, Ali, SMM, and Memon, TU. Waveguide photodetector for optical integrated circuits. IEEEP Multitopic International Symposium-97, Karachi, March 5-6, 1997.

6. Hunsperger, R.G, Yariv, A, and Lee, A. Parallel end-butt coupling for optical integrated circuits. // Applied optics; 16(4), 1026-1032 (1977).

7. Alping, A, Tell, R, and Eng, S.T., Photodetection properties of semiconductor laser diode detectors. // J. of Lightwave Technol.; LT-4(11), 1662-1668 (1986).

8. Hasnain, G, Tai, K, Wang, Y.H., Wynn, J.D., Choquette, K.D., Weir, B.E., Dutta, N.K., and Cho, A.Y. Monolithically integration of photodetector with vertical surface emitting lasers. // Electron. Lett.; 27(18), 1632-1632 (1991). 